\title{
Médecins, chirurgiens, apothicaires : à qui sont adressées les traductions médicales? Enquête sur l'édition lyonnaise des années 1540
}

\author{
ÉLISE RAJCHENBACH \\ Université de Lyon \\ Université Jean-Monnet Saint-Étienne \\ IHRIM — UMR 5317
}

\begin{abstract}
Dans les années 1540, l'édition médicale est en vogue à Lyon, en particulier à travers les traductions de Galien. Or, les préfaces des traductions du médecin grec mettent en lumière les conflits qui soustendent pour les médecins, chirurgiens et apothicaires la pratique de leurs domaines voisins. Traduire Galien et ses épigones revient en effet à rendre accessible la science antique, jusqu'alors réservée aux lecteurs de latin, voire de grec, c'est-à-dire aux savants. Nous étudions les enjeux qui président à la diffusion de ces traductions ainsi que les réceptions qu'elles semblent avoir entraînées auprès d'un public plus polymorphe qu'il n'y paraît, dans un contexte plus vaste de réflexion sur le rôle de la traduction comme vecteur de diffusion de savoirs.
\end{abstract}

In the 1540s, medical publishing is in fashion in Lyon, specifically in the form of translations of Galen. Prefaces to the translations of the Greek physician highlight the conflicts underlying the practices of doctors, surgeons, and apothecaries in their closely-related disciplines. Translating Galen and his successors actually amounts to making accessible ancient science which was until then reserved for readers of Latin or Greek, that is to say, scholars. We study the issues that oversee the circulation of these translations as well as the receptions they enabled among a reading public much more diverse than it might seem, within a larger context of reflection on the role of translation as a vehicle for the dissemination of knowledge.

$\mathrm{C}_{\text {traductions en français de Galien dans sa formation : }}^{\text {n 1550, Ambroise Paré, futur chirurgien du roi, souligne l'importance des }}$

Je ne veulx m'arroger que j'aye leu Galien parlant grec, ou latin. Car n'a pleu à dieu tant faire de grace à ma jeunesse, qu'elle aye esté en l'une et l'autre langue instituée. Mais aussi ne vouldroys aucunement dissimuler, que j'ay apris lesdictz documents de Galien par l'interpretation françoyse 
de monsieur maistre Jehan Canape, docteur regent en la faculté de médecine faisant sa demeurance à Lyon. ${ }^{1}$

Médecin lyonnais, ancien régent du Collège de la Trinité à Lyon — fonction dont il a démissionné en 1530 - Jean Canappe appartient à la promotion 1530 de l'université de Montpellier². Il est l'un des principaux pourvoyeurs, sur la place lyonnaise (et plus largement française) des traductions médicales de la fin des années 1530 et du début des années 1540. À cette entreprise se joint bientôt son ami Pierre Tolet, formé également à Montpellier ${ }^{3}$. On peut également formuler, à la suite de Roland Antonioli, l'hypothèse d'une participation de Rabelais aux premières traductions, en particulier à celle du Cinquiesme livre 4 . À ces traductions de Galien s'adjoignent d'autres éditions et traductions, toujours par les deux mêmes médecins, de textes médicaux fondés pour une large part sur l'enseignement galénique.

Le milieu des traductions médicales, qui tournent essentiellement - et logiquement - autour de Galien et de ses successeurs, est très cohérent à Lyon. C'est un petit groupe qui se retrouve en collaboration étroite avec les ateliers d'imprimeurs : François Juste (pendant vernaculaire de l'atelier de Gryphe), puis Étienne Dolet, qui collabore régulièrement avec le libraire Guillaume de Guelques, l'association transitoire entre Constantin et Sabon, et enfin Jean de Tournes.

D’après Richard Durling 5 , qui répertorie les éditions et traductions de Galien en Europe entre 1473 et 1600, la première traduction en français de Galien est celle du Quatriesme livre de la therapeutique de Galien par Jean

1. Ambroise Paré, Briefve collection de l'administration anatomique : avec la maniere de conjoindre les os: Et d'extraire les enfans mors que vivants du ventre de la mere, lors que la nature de soy ne peult venir à son effect. Composé par Ambroise Paré maistre Barbier Chyrurgien à Paris (Paris : Guillaume Cavellat, 1550), fol. A $6 \mathrm{r}^{\circ}$.

2. En 1542, il obtient le poste de médecin de François I ${ }^{\mathrm{er}}$. Voir Marion Bertin, Le Collège de la Trinité : histoire d'une bibliothèque et de son Cabinet de Curiosités (Mémoire de master 1, École nationale supérieure des sciences de l'information et des bibliothèques, juin 2014), 18.

3. Il appartenait à la promotion précédente de 1529, où l'on retrouve Rabelais, Jacques Sylvius, Rondelet ou Nostradamus. Voir Roland Antonioli, Rabelais et la médecine (Genève : Droz, 1976), 39.

4. Antonioli, Rabelais et la médecine, 100, au sujet du Cinquiesme livre de la methode therapeutique de Claude Galien (Lyon : François Juste, 1537).

5. Richard Durling, "A Chronological Census of Renaissance Editions and Translations of Galen ", Journal of the Warburg and Coutauld Institutes 24.3 (Jul.-Dec., 1961) : 230-305. 
Canappe, publiée à Lyon chez François Juste en $1537^{6}$. La traduction de Canappe est suivie d'une série d'autres traductions dès l'année suivante, rapidement prises en charge par Dolet, d'abord comme éditeur, puis comme imprimeur, rôle qu'il expose dans l'avant-propos de l'édition du Troisiesme livre publié par Guillaume de Guelques :

Etienne Dolet à Maistre Jehan Canappe Docteur en Medecine Salut. Sache, Amy, que l'utilité que j'ay congneu proceder des Livres de Galien : c'est assavoir le III, IIII, V, VI, XIII, et XIIII, de la Methode Therapeutique, avec le Second de l'Art Curatoire a Glaucon lesquelz m’as baillé, sur foy de les mettre fidelement en lumiere, m'a induit (avec l'amytie, que je te porte) d'y vaquer en la sorte, que telz CEuvres requierent. Et soubz le Privileige, que le Roy m'a donné, maintenant sortent en lumiere. Prendz donc en gré mon labeur : et ne te lasse en ton endroict, de proffiter au bien public Literaire. de Lyon ce XXV de Janvier 1539.

Dans un très riche article paru dans les actes du colloque consacré à Étienne Dolet, Valerie Worth-Stylianou a étudié les publications médicales de Dolet en montrant qu'elles entraient en concordance avec les préoccupations

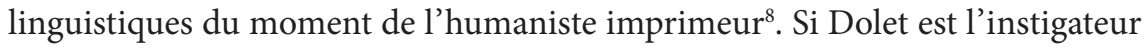
des publications de traductions médicales à Lyon (et en France), le corpus est toutefois plus vaste car les traducteurs poursuivent leur œuvre au-delà de la mort de Dolet. Surtout, nous aimerions adopter ici un point de vue qui ne parte pas de l'imprimeur-libraire, mais d'un corpus constitué par les traductions en français des textes médicaux à la fin des années 1530 et dans les années 1540, en étudiant les dédicaces de ces traductions : plus polymorphe qu'elle n'y paraît, la nature des lecteurs envisagés par les traducteurs rend compte de tensions et dynamiques qui agitent le milieu médical, et plus largement humaniste, de ces années.

6. Le quatriesme livre de la Therapeutique ou methode curative de Claude Galien, prince des Medecins, auquel est singulierement traictée la cure des ulceres, translaté par Philiatros (Lyon : François Juste, 1537).

7. Le Troisiesme Livre de la Therapeutique ou Methode curatoire de Claude Galien prince des Medecins, auquel est singulierement traictée la cure des Ulceres. Avec privileige pour VI. Ans (Lyon : chez Guillaume de Guelques, [1539]), fol. a $1 \mathrm{v}^{\circ}$.

8. Valerie Worth-Stylianou, "Étienne Dolet et l'édition médicale », in Étienne Dolet. 1509-2009, éd. Michèle Clément (Genève : Droz, 2012), 361-379. 


\section{Une nécessaire formation des chirurgiens}

Parmi les quatorze ouvrages dépouillés ${ }^{9}$, dix présentent une dédicace du traducteur. Le destinataire est, pour la grande majorité des paratextes ${ }^{10}$, un « lecteur » qui n'est pas d'emblée caractérisé mais qu’on identifie généralement assez rapidement, au premier abord, à un chirurgien. Trois autres dédicaces sont explicitement adressées au « lecteur chirurgien ». Ce public désigné n’a rien de surprenant : contrairement aux médecins, qui étudient le grec et le latin, les chirurgiens ne pratiquent pas les langues dans lesquelles sont publiés les médecins grecs, ce qui rend leur formation passablement incomplète. Or, ce problème d'accès aux textes est créé ou plutôt renforcé par une conception particulièrement hermétique des différentes parties de la médecine dont rend compte Jean Canappe :

Toutesfois aujourdhuy [...] on separe lesdictes parties de medecine, desquelles la premiere est demeurée a ceulx que le vulgaire appelle medecins. La seconde aux apoticaires, dont ilz retiennent le nom des pharmacopoles. Et la tierce est demeurée aux chirurgiens. Tellement que aujourdhuy le medecin se fie du tout ou a peu pres aux apoticaires, en la congnoissance des simples medicamens : laquelle est si necessaire, qu'on ne scauroit bien composer, ne bien user des medicamens jadis composez sans icelle. ${ }^{11}$

Jaloux de leur art et de leurs prérogatives, les médecins s'opposeraient ainsi, par une sorte de privilège de langue et de classe, au partage de larges pans du savoir médical, ce qui n'est pas sans danger :

Esquelles operations, si tu ne congnoys la situation du nerf, ou muscle principal, de la grande artere, ou veyne, tu seras aulcunesfois autheur, et cause plustost de la mort, que de la santé des malades. Et aulcunesfois tu en feras les ungs manchets, les aultres boyteux. ${ }^{12}$

9. Voir annexe. On y trouvera les références complètes des ouvrages cités dans les notes suivantes.

10. Sept dédicaces.

11. Jean Canappe, "Philiatros au Lecteur Salut ", in Le quatriesme livre de la Therapeutique (Lyon : François Juste, 1537), f. 2 r .

12. Jean Canappe, «Le translateur au lecteur », in Du Mouvement des muscles, fol. F $3 \mathrm{v}^{\circ}$. 
Les paratextes de nos traductions soulignent tous la nécessité de former les chirurgiens, afin qu'ils puissent exercer leur art dans les meilleures conditions, en secondant efficacement le médecin sans tuer le malade. Un barbier mal instruit peut s'avérer fort dangereux pour le malade, plus, même, que la maladie. Justifiant la mise à disposition de la science anatomique pour les chirurgiens, Canappe assimile ainsi, dans une métaphore percutante, à la suite de Guy de Chauliac, le chirurgien et le bûcheron aveugle. Un chirurgien ne maîtrisant pas l'anatomie serait comme un bûcheron aveugle frappant au hasard:

Or la fin de l'art, que nous pretendons, est de garder la santé, et de guerir les maladies : à laquelle nul ne peult parvenir, s'il ne sçait, que c'est santé, ou maladie. Et pource que toute santé, ou maladie consiste, ou aux parties simples, ou aux parties organiques, ou est commun à icelles, il s'ensuit, qu'il fault congnoistre lesdites parties. A la congnoissance desquelles n'avons aucune voye, ny adresse sinon par l'anatomie. [...] Par l'ignorance desquelles choses, quand un nerf est incisé, ou un muscle, il advient aucunes fois, que le sentiment se perd, aucunesfois le mouvement, et souventesfois l'un, et l'autre, et qui plus est, bien souvent la mort s'ensuit. Chacun voit donc la grande, et necessaire utilité de l'anatomie : laquelle est si requise en aucun, c'est principalement au Chirurgien. Et combien que la chose soit assez attestée, toutesfois je allegueray encores ce petit mot digne de memoire, que dit Guidon de Cauliac: Ainsi comme l'aveugle, qui trenche du boys, tousjours, ou souvent erre, en trenchant d'iceluy plus, ou moins, qu'il ne doit : ainsi fait le Chirurgien, quand il ignore l'anatomie. ${ }^{13}$

Cette nécessité de connaître l'anatomie pour exercer proprement la chirurgie est un lieu commun de l'argumentation de Canappe qui ne cesse de la réaffirmer depuis ses premières traductions ${ }^{14}$. Stratégiquement, Canappe se fonde sur l'autorité incontestable de Galien et d'Hippocrate, qui revendiquent la perméabilité des disciplines médicales:

13. Jean Canappe, « Maistre Jean Canappe Docteur en Medecine au Lecteur Chirurgien Salut », in Tables anatomiques $d u$ corps humain universel, 4-5.

14. Par exemple, Jean Canappe, "Le translateur aux Chirurgiens Salut ", in L'Anatomie des os du corps humain: "Congnoissant que la chirurgie ne se peult exercer, comme il appertient, sans l'anatomie, pour ceste cause j'ay voulu traduire ce present livre, comme celluy, qui doibt estre le principe, et fondement de l'anatomie». 
Et quand a la chirurgie (qui n'est sinon manuelle operation) les medecins l'estiment une chose trop vile, et indigne de leur profession, et non seulement ladicte manuelle operation, laquelle Hipocrates et Galien n'ont eu honte de traicter, et exercer, mais aussy la methode de curer les ulceres, et tumeurs contre nature a esté par eulx delaissée : en sorte que les barbiers et chirurgiens en sont aujourdhuy plus studieux que aulcuns medecins. ${ }^{15}$

En ce sens, le traducteur affirme s'adresser à un public de chirurgiens avides d’apprendre ce qui leur était jusqu'alors caché.

Canappe souligne régulièrement que c'est à la demande, parfois pressante, des chirurgiens qu'il traduit et publie. Répondant à une forme d'urgence, il officie presque sur commande, pour le bien public:

Quant au neufviesme livre des simples, je ne pensoye pas y mettre la main, car j'avoye entendu, qu'il estoit ja traduict, il y a des ans plus que troys, par quelq'ung, lequel possible le veult produyre en la maniere des Elephantz. Ou bien veult user du conseil de Horace : lequel suade en son art poëticque, de ne point precipiter ung oeuvre : ains le garder neuf ans. Possible, que le neufviesme an, il esperoit parachever le dict neufviesme livre des Simples : c'est environ ung feuillet pour an, qui est une belle besongne, et digne de grande reputation. Or plusieurs Chirurgiens (pour la promesse à eulx faicte) attendoyent de jour en jour, que ce dict livre vint en lumiere. Mais d'aultant, que la longue comperendination, et delay les frustroit de leur

15. Canappe, «Philiatros au Lecteur Salut », f. $2 \mathrm{r}^{\circ}$. Voir également, par exemple, du même auteur, "Le translateur au Lecteur, Salut ", in Le tresiesme Livre de la Methode Therapeutique, f. $103 \mathrm{v}^{\circ}-104 \mathrm{r}^{\circ}$ : "Car d'autant que l'art de medecine est divisée en troys parties, l'une ne peult estre separée de l'autre, sans le dommaige et grand detriment de toute la profession medicinale, ainsi que nous avons plus a plain demonstré en l'epistre liminaire du quatriesme livre, par ceste raison la consideration et congnoissance des temperatures et intemperatures n'est pas impertinente aux chirurgiens methodiques. Car aussi comme Galien dict tant souventesfoys, en disputant contre Thessalus et ses disciples, L'on ne sçauroit curer par methode (c'est a dire voye rationale, une playe, ou ulcere, ou fracture, sans sçavoir la nature des parties du corps humain [...]. Neantmoins pource qu'il se fault accommoder a la coustume du pays, ainsi que faysoit Galien, lequel comme il recite au sixiesme livre de la methode, ensuyvant la maniere de la cité de Rome, en permettant la curation des fractures aux chirurgiens ». Canappe propose un développement très proche dans l'épître « Ioannes Canapæus Guilielmo Rondeleto Monspessulano Medicao S. ", in Du Mouvement des muscles, 6, repris au début de l'épître au lecteur, en français, qui figure à la fin du même ouvrage. 
expectation, m'ont efflagité, et quasi par quotidien convice contrainct, de prendre ce labeur de leur traduyre le dict neufviesme livre des Simples. Ce que j'ay faict $[\ldots]^{16}$

Par la référence ironique à Horace, Canappe disjoint violemment l'art poétique et la pratique médicale. Il extrait la pratique des barbiers et autres apothicaires de la poétique et de la rhétorique pour lui rétablir son utilité immédiate. Cette idée réapparait dans la revendication de l'usage du français :

Considerant, que l'art de medecine, et chirurgie ne gist pas du tout aux langues. Car c'est tout ung de l'entendre en Grec, ou Latin, ou Arabic, ou Francoys, ou (si tu veulx) en Breton Bretonant, pourveue qu'on l'entende bien. Jouxte la sentence de Cornelius Celsus, lequel dict que les maladies ne sont pas gueryes par eloquence, mais par remedes. ${ }^{17}$

Ne confondons pas les disciplines : c'est le contenu et non la langue dans lequel le premier est transmis qui compte. Canappe rejette ainsi les réticences, voire les blocages des médecins vis-à-vis de toute instruction des chirurgiens et repousse leurs objections. Car derrière le premier public visé dans les dédicaces et dans le fait même de traduire en français, se profile un autre public, moins acquis, celui des détracteurs qui ne manqueront pas de lire une traduction décriée et qui font face aux médecins progressistes désireux de former les chirurgiens pour le bien commun. Cet autre public des traductions ne se définirait plus par le contenu des traductions mais par la démarche qu'elles incarnent. Traduire et publier des textes médicaux en français, c'est prendre position dans un débat.

\section{Les médecins : commanditaires et public second des traductions médicales ? Un public en conflit}

Les traductions de Galien en français semblent s'adresser à un public plus large qu'il n’y paraît. Dans sa dédicace «Au lecteur » qui précède sa révision du Guidon de Chauliac, Jean Canappe présente ainsi les solliciteurs qui l'ont incité, avec insistance, à publier le texte :

16. Jean Canappe, «Le translateur au lecteur Salut », in Deux livres des simples de Galien, fol. A 3 v$^{\circ}$

17. Canappe, «Le translateur au lecteur», fol. F 4 v. 
Lecteur docile et de bon vouloir, Je requis et souventesfois sollicité par plusieurs de mes amys, et mesmement par ceulx qui versent continuellement a l'estude et exercice de l'art de Chirurgie, en ceste Insigne et celebre cité de Lyon, tant en mon auditoire, que aussi des autres excellens et eminentissimes Docteurs en Medecine en ladicte Ville : me suys mis a mon debvoir, selon la petite faculté et intelligence qu'il a pleu a Dieu me donner, de recongnoistre et emender la Chirurgie de Maistre GUidon de Cauliac en son temps excellent Chirurgien et Docteur en Medecine en la tresamée université de Montpellier. ${ }^{18}$

À l'auditoire des chirurgiens avides d'accéder à un texte de référence, le Guidon, s’adjoignent les médecins. L’ancrage lyonnais affiché par Canappe dessine les contours - largement fictifs - d'une communauté médicale unie par un désir commun, une édition fiable du Guidon qui permettrait une pratique harmonieuse et efficace de toutes les parties de la médecine. Ce refus de séparer les deux communautés est confirmé par la présentation de l'auteur, à la fois « excellent Chirurgien et Docteur en Medecine ». Chauliac unit ainsi deux arts traditionnellement distingués, tant dans sa personne que dans son public. Et de manière remarquable, en unissant ces deux communautés dans sa dédicace au lecteur, Canappe s'adresse à un public polymorphe qui ne se réduit pas aux simples barbiers ou chirurgiens qui, à la différence des médecins, ne peuvent lire que le français. L'entreprise de traduction ne restreint pas, selon Canappe, le champ des destinataires, mais il l'élargit et en unit les composantes autrefois disjointes. Il ne s'agit pas pour autant de nier ou de rejeter la différence des arts, mais de revendiquer une certaine perméabilité des champs du savoir qui permette à chacun d'exercer son art avec efficacité et sûreté. Canappe ne prétend pas abattre les murs entre les différents arts de médecine, mais offrir les outils qui permettront à chacun d'exercer le sien proprement, en conservant sa spécialité, en accord et en complémentarité avec l'autre. La traduction se présente alors comme une entreprise de réconciliation idéale de publics jusqu'alors désunis.

En ce sens, on trouve dans le texte des dédicaces des passages particulièrement adressés, directement ou indirectement, aux médecins. Il s'agit, dans le corps du texte, d'accusations contre les médecins refusant le 18. Jean Canappe, "Maistre Jehan Canappe, au lecteur salut », in Le Guidon en Francoys nouvellement reveu, et au vray corrigé, fol. a $2 \mathrm{r}^{\circ}$. 
partage des savoirs, rendues parfois plus visibles sous forme de manchettes. Dans la dédicace du Mouvement des muscles, la première des deux manchettes stigmatise "L'envie de aucuns medecins" :

Or ce n'est pas de maintenant, que regne ceste envie, ou plustost (si j’ausoye dire) ceste avarice, et insatiable cupidité d'aulcuns. Je croy qu'ilz ont peur, qu'on ne sache trop, ou autant, et plus, que eulx : ou plustost que terre ne leur defaille. Je ne scauroye (à mon advis) mieulx comparer telle maniere de gens, que à celluy, qui avoit receu le talent de son maistre, et puis le cacha dans la terre, sans en faire aulcun proffit. Ou à celluy, qui a une lumiere, laquelle il va chacher [sic] dessoubz ung muy, à celle fin, que les aultres n'y veoyent rien. Ou finablement à celluy, qui a la clef pour entrer en quelque lieu, toutesfois il n'y veult entrer, ne laisser entrer les aultres. $^{19}$

Canappe tend à son lecteur un contre-miroir dans lequel il présente à la troisième personne un médecin avaricieux à l'insatiable cupidité - et, somme toute, mauvais chrétien. Et le texte s'achève par un glissement qui, par le retour à la deuxième personne, permet d'identifier le lecteur non comme chirurgien mais bien comme médecin appelé à accepter ce travail de traduction : « Te suppliant lecteur ne te vouloir fascher de nostre labeur, jusques à ce que tu te repentes, et estimes peu de chose de proffiter en ton art $»^{20}$. Au contraire de ce contre-miroir du médecin avaricieux, Canappe propose le miroir de Galien, qui n'a eu de cesse de partager son savoir et dans le sillage duquel s'inscrit le traducteur:

Item consequemment dict ledict Galien, qu'il a departy, et communicqué avec une candeur, et franc vouloir, tout ce qu'il scavoit, à ceulx qui l'en requeroient : desirant, que tout homme en eust congnoissance, tant que possible seroit. Jacoit qu'il entendist bien, que plusieurs de ses disciples ne vouloient pas communicquer leur scavoir aux aultres : laquelle chose (comme il dict) ne procedoit, que d'envie $[\ldots]^{21}$

19. Canappe, "Le translateur au lecteur », fol. F $3 v^{\circ}$.

20. Canappe, «Le translateur au lecteur », fol. F $3 v^{\circ}$.

21. Canappe, "Le translateur au lecteur », fol. F 3 r $^{\circ}$. 
Face à ces mauvais médecins se dressent deux figures nobles, dont le nom apparaît dans les dédicaces respectives de deux traductions : Jean Schyron et Guillaume Rondelet. Pierre Tolet fait figurer deux paratextes en tête de sa traduction de la Chirurgie de Paulus Egineta"22 : une dédicace "A Monsieur Squironis [...] » et un "Proeme au Chyrurgien Francoys ». Jean Schyron, présenté comme « docteur Royal en l'université de Montpelier, et Medecin de la Royne de Navarre » a été l'un des maîtres de Pierre Tolet ${ }^{23}$, comme ce dernier le rappelle dans son édition de Paulo Bagellardo en $1538^{24}$. Schyron, helléniste de qualité, est arrivé à Montpellier en 1526 et marque profondément ses disciples par le combat qu'il mène contre l'ignorance et le manque de pratique. Scaliger le raille, dans ses Poemata, stigmatisant le fait qu'il contrôlerait, voire élaborerait lui-même les médicaments qu'il enseigne ou qu'il prescrit ${ }^{25}$. Conformément à l'orientation de l'université de Montpellier, Schyron défend en effet un savoir qui s'appuie fermement sur l'expérience, conviction qui soutient la construction de l'amphithéâtre d'anatomie peu avant sa mort en 1556. Rappelons à cet égard que dès 1514 , l'enseignement pour les chirurgiens

\section{La chirurgie de Paulus Aginata (Lyon: Dolet, 1540).}

23. Sur Jean Schyron, voir Antonioli, Rabelais et la médecine, 39-47.

24. Opusculum recens natum de morbis puerorum cum Appendicibus Magistri Petri Toleti ex professo Medici. Sunt etiam nonnulli additi Libelli perutiles, hactenus desiderati, quos sequens pagella demonstrabit (Lyon : Germain Rose, 1538), 113 : «Schironius etiam præceptor meus Archiatros, non Aquitaniæ modò, set et Galliarum, cuius ego uerbum non facio sine admiratione et laude ". "Schyron, mon maître, dont moi-même je ne parle pas sans admiration ni estime, est également médecin royal non seulement en Aquitaine mais aussi dans les Gaules " (toutes les traductions sont nôtres, sauf indication spécifique). L'épilogue de Tolet à l'« Additio quorundam morborum de quibus nihil est loquutus autor iste ", 110-115, propose une réflexion fondamentale sur la transmission des textes grecs et le risque constant de leur disparition.

25. Voir l'épigramme des Poemata que Scaliger (Manes Catulliani, I, 639) adresse à un certain Calvus, surnom injurieux de Jean Schyron, cité par Antonioli, Rabelais et la médecine, 38 « Ad montem Pessulum de Calvo »: « Ne exitio et fame periret / Factus clinicus ipsemet coquinis, / Pinsit ptissanam et rota verutum ; / Rimatur luteum foramen ani / Quo clysteria tergimerda condat ». C'est Jacques Scaliger, dans les Scaligerana, qui identifie Calvus à Jean Schyron : "Scirrhonius, ignarissimus vir, pharmacotriba, id est Pileur de drogues, verius quam medicus. Is est Calvus ille, carminibus patris Julii Scaligeri decantatissimus cuique stigmata inussit ", cité d'après l'édition de Cologne, 1595, 364, par le Dr de Santi, «Rabelais à Toulouse », Revue du Seizième siècle 1-2 (1921) : 47, n. 2 («Schyron était un homme très ignare, vraisemblablement plus pileur de drogues que médecin. C'est ce Calvus qui apparaît constamment dans les poèmes de mon père Jules Scaliger, et sur qui ce dernier a imprimé au fer rouge des marques d'infamie»). 
dans les écoles de Montpellier est dispensé en français (contre 1576 seulement, à Lyon) et les futurs médecins bénéficient d'une lecture et d'un commentaire de la chirurgie de Chauliac, comme ceux qui sont effectués par Jean Falcon en 1527 en latin ${ }^{26}$. En dédiant ses traductions de Paul d'Égine et de Galien à Jean Schyron, Pierre Tolet légitime sa démarche en l'inscrivant dans une tradition médicale illustre et surtout à l'avant-garde - quoique débattue et décriée par certains. Tolet dessine les contours d'un public double, celui des barbiers - il évoque d'emblée " la continuelle priere (pour leur necessité, et usage) des compaignons Chyrurgiens de la ville de Lyon ${ }^{27}$ - et celui des médecins. L'identité de l'interlocuteur glisse insensiblement de Schyron au médecin en général :

Aussi toy, qui es personne raisonnable (je parle aux Detracteurs, et a ceulx, qui veulent la congnoissance des artz estre cachée) comment veulx tu, que ton serviteur fasse ton commandement, et entende, ce que tu dis, par langue incongneue ? qui est autant que d'estre muet. Tu veulx, que le Chirurgien de maintenant n'entende point son art, et qu'il soit bon operateur, et moindre que toy en scavoir : ce qui ne se peult faire..$^{28}$

Si la traduction des médecins grecs est d'abord identifiable comme un outil de formation nécessaire pour les chirurgiens, c'est également, nous semble-t-il, le support d'une revendication dans le cadre d'un débat plus vaste qui agite le milieu médical, celui de la querelle arabe qui a battu son plein au cœur des années 1530. Et de ce point de vue, le public de la série des traductions lyonnaises des textes galéniques (et hippocratiques) est bien constitué par les médecins qui seront les premiers bénéficiaires d'un abandon des pratiques empiriques et superstitieuses héritées, selon ses détracteurs (au premier chef Champier à Lyon), de la médecine arabe. L'évocation, par Tolet, du travail de traducteur et de commentateur de Canappe, est ainsi l'occasion de dresser un nouveau contre-miroir qui va de pair avec le maintien condamnable et dangereux de la science médicale dans le secret latin :

26. Antonioli, Rabelais et la médecine, 38.

27. Pierre Tolet, «A Monsieur Squironis docteur Royal en l'université de Montpelier, et Medecin de la Royne de Navarre, Maistre Pierre Tolet docteur en Medecine, humble Salut ", in La chirurgie de Paulus Eginata, 3.

28. Tolet, « A Monsieur Squironis », 6-7. 
En cest endroict suis contrainct par raison, et verité, de louer par sus touts ung medecin Lyonnois appellé Maistre Jehan Canappe : auquel (comme je pense) la Chyrurgie doibt plus qu'à homme, qui ayt escript depuis Galien : duquel il interprete les Oeuvres pour ton usage, et proffit : n'abusant le monde, comme ung tas de Lanterniers advolés en France de plusieurs païs estranges : lesquelz (au grand regret des gens scavantz) abusent le pauvre populaire Francoys : les ungs faisant profession de magicque : les aultres s'abillant (pour admiration) maintenant de blanc, maintenant de verd, maintenant de gris, maintenant de rouge. Ò quelle ignorance de peuple, de se laisser tromper par telles bestes, et abuseurs tant manifestes. Desquelz abus la fin est de faire ung malade (tombant entre leurs mains) ou à jamais languissant, ou bien tost mort. ${ }^{29}$

Diffuser la science galénique contre l'empirisme approximatif arabe, c'est permettre au chirurgien comme au médecin d'exercer son art dans de bonnes conditions, sans avoir à affronter la concurrence des charlatans et marabouts en tous genres, susceptibles de faire revenir l'être aimé la queue entre les jambes et de guérir la syphilis par l'emploi de poudre de perlimpinpin. C'est défendre aussi la noblesse de l'art médical - le vrai - et combattre la crédulité populaire. En ce sens, derrière le public des chirurgiens désireux d'exercer efficacement leur art se dessinerait un autre public, celui des médecins pris dans la querelle arabe qui est présentée avant tout comme une querelle contre l'ignorance. L'existence de ces traductions - qui ne seront peut-être pas lues en tant que telles par les médecins - fonctionne comme une prise de position au sein du débat : elle est une forme d'acte, en soi, qui s'adresse bien, cette fois, aux médecins.

Si Tolet se fonde sur l'autorité de son professeur de l'université de Montpellier, Canappe n'est pas en reste, puisque c'est à l'un de ses condisciples qu'il adresse sa traduction du Mouvement des muscles en 1541, Guillaume Rondelet $^{30}$, issu de la promotion de 1529 et reçu docteur en 1537 à Montpellier. Savant touche-à-tout, il se fait également connaître, plus tard, pour ses travaux d'ischiologie et de botanique. La longue épître de six pages qui lui est adressée figure en tête du traité du Mouvement des muscles et repousse l'« Epistre du Translateur au Lecteur » en fin de volume. Elle est écrite en latin et constitue, par

29. Pierre Tolet, " Proesme au Chyrurgien Francoys », in La chirurgie de Paulus Ægineta, 14-15.

30. Canappe, « Ioannes Canapæus Gulielmo Rondeleto Monspessulano Medico s. », 3-8. 
le choix même de la langue, un paradoxe apparent. Après un long passage qui revendique l'usage de la langue maternelle plutôt que d'une langue étrangère, Canappe souligne que la traduction présentée ici n'est pas pour les médecins mais pour les chirurgiens ${ }^{31}$. Toutefois, la présence de cette lettre latine, qui reprend des développements déjà déployés dans des paratextes français, met en lumière ce double destinataire dont la silhouette se dessinait déjà chez Tolet. Symboliquement, Canappe, réunit les deux langues et les deux milieux autour d'une figure qui incarne l'ouverture montpelliéraine.

\section{L'adresse à un public humaniste}

L'épître à Rondelet, par le discours qu'elle déploie en faveur de l'emploi de la langue française, rappelle assez nettement l'aboutissement des positions cicéroniennes de Dolet, avec qui Canappe est très lié au début de son entreprise de traduction, comme l'a montré Valerie Worth-Stylianou : "Cur enim alienam, ac peregrinam sectabimur linguam, ut nostram deseramus ? $»^{32}$. Pourquoi avoir recours à une langue étrangère, alors que, finalement, nos auteurs antiques ont quant à eux eu recours à leur langue pour transmettre leur savoir ? Si Galien avait pour constant objectif de partager ses connaissances médicales, de même, ses traducteurs en français combattent l'ignorance. En ce sens, nos traducteurs inscrivent l'approche montpelliéraine de la médecine dans la dynamique plus vaste de diffusion humaniste des savoirs antiques. Canappe en particulier souligne le rôle de transmission qu'il endosse afin de parer le risque constant de disparition des savoirs.

Et pource qu'il y a dangier, que icelles speculations ne perissent, tant à cause de la negligence des hommes environ les artz, et sciences, que aussi

31. «Ego tibi non uerti, neque doctis omnibus (in quorum albo te esse desiderarem) sed linguæ utriusque imperitis, cuiusmodi est bona pars chirurgorum huiusce tempestatis : quibus maiorem eruditionem bene precor » (Canappe, « Ioannes Canapæus Gulielmo Rondeleto Monspessulano Medico s. », 5) : "Pour ma part, ce n'est pas pour toi que j'ai traduit, ni pour tous les savants (au nombre desquels je souhaiterais que tu sois compté), mais pour ceux qui sont ignorants de l'une et l'autre langue [grecque et latine], auquel genre sont comptés une bonne part des chirurgiens de ce temps-ci : pour lesquels j'appelle bien de mes vœux un plus grand savoir ».

32. Canappe, «Ioannes Canapæus Gulielmo Rondeleto Monspessulano Medico s. », 3 : « Pourquoi en effet poursuivons-nous une langue étrangère et pérégrine, en sorte que nous négligeons la nôtre ?». 
à cause qu'il [sic] n'ont pas accoustumé d'y estre exercité de jeunesse, il est expedient faire des livres, et commentaires. ${ }^{33}$

Si un savant médecin vient à mourir sans avoir transmis son savoir, ce dernier serait définitivement perdu ${ }^{34}$. Traduire devient ici un devoir éthique :

C'est que nous ne sommes pas seulement naiz pour nous, mais que nostre païs, et noz amys doybvent avoir quelque partie de nostre naiscence : c'est à dire quelque fruict de nous. Voyla l'occasion laquelle m'a induict (selon ma promesse) de donner quelque entrée, et intelligence en l'anatomie, tant des os, que des muscles, et de leur mouvement, à ceulx qui ne sont aulcunement institués es langues, Grecque, ou Latine. ${ }^{35}$

Canappe prend à témoin de ce devoir son lecteur, dont l'identité devient flottante :

Et à telz j'escris, et pour telz je travaille, non pour les doctes, et sçavans, qui n'ont aucunement affaire de ce mien effort. Toutesfois je croy, qu'il n'y ha sçavant, ou docte, qui ne trouve bonne ceste mienne entreprinse, attendu la fin d'icelle, qui est de profiter au public. Or donc, Lecteur, quiconque sois, prens en gré le vouloir honneste de celuy, qui ne desire, que ton avancement, et l'augmentation de ton sçavoir, et te jetter peu à peu des tenebres d'ignorance $[\ldots] .{ }^{36}$

Au-delà du lecteur chirurgien, premier bénéficiaire, dans le domaine pratique, de cette traduction des tables anatomiques de Vassé et par conséquent de la mise à disposition d'un savoir anatomique jusqu'alors réservé aux médecins, Canappe esquisse une autre silhouette de lecteur, humaniste cette fois, sensible à l'entreprise de diffusion des savoirs endossée par le traducteur médecin. La traduction médicale permet de combattre l'ignorance et le traducteur se

33. Canappe, «Le translateur au lecteur", in Du Mouvement des muscles, fol. F $3 \mathrm{r}^{\circ}$.

34. Canappe, «Le translateur au lecteur », in Le Mouvement des muscles, fol. F $3 \mathrm{r}^{\circ}$ : «Lesquelz si venoient à mourir subdainement, leur scavoir seroit perdu ».

35. Canappe, «Le translateur au lecteur », Le Mouvement des muscles, fol. F $4 \mathrm{r}^{\circ}$.

36. Canappe, "Maistre Jean Canappe Docteur en Medecine au Lecteur Chirurgien Salut ", in Tables anatomiques du corps humain universel, 7. 
trouve portraituré en médecin de l'âme : «ignorance indigne de tout homme genereux, et bien nay : laquelle devons autant, ou plus, fuyr, que la mort. Car l'homme ignorant n'est autre chose, que une mort vive [...] ${ }^{37}$. À la faveur du parallèle ainsi établi et par un certain coup de force, le médecin est assimilé, en filigrane, à l'humaniste dont la raison d'être est de diffuser les savoirs et de combattre l'ignorance. Canappe cultive l'assimilation des matières médicales et humanistes. Il entame ainsi l'épître de « Philiatros au Lecteur » qui ouvre Le quatriesme livre de la Therapeutique de Galien par un développement sur les liens étroits qui unissent philosophie et éloquence :

\begin{abstract}
Amy lecteur, Quintilian en son premier livre de l'institution oratoire recite comment philosophie et eloquence sont conjoinctes par nature, et unies ensemble par office et action. Neantmoins l'estude de philosophie et eloquence a esté separé l'un de l'autre, tellement que la negligence des hommes a faict qu'ilz semblent estre plusieurs ars, et sciences diverses. Et rend la raison pourquoy : Car depuis que la langue et eloquence a commencé de s'adonner à la practique, je dis lucrative exercitation, et qu'on a abusé des biens et graces d'eloquence, on a abandonné et du tout delaissé la cure des vertus, et bonnes meurs : qui est la vraye philosophie : laquelle de sa propre nature doit estre conjoincte à eloquence. ${ }^{38}$
\end{abstract}

Philosophie et éloquence ont subi une distinction forcée entre théorie et pratique, de telle sorte qu'elles ont perdu leur raison d'être. C'est le même dévoiement qui a affecté médecine et chirurgie :

En suyvant la sentence de Quintilian, je dis semblablement que les parties
de l'art de medecine, c'est assavoir dietetique, pharmaceutique, et chirurgie,
sont tellement compliquées et connexées ensemble, que nullement ne
pourroient estre separées l'une d'avec l'autre, sans le dommaige, et grand
detriment de toute la profession medecinale. Car l'une est aydée, parfaicte,
et consommée par les autres, en sorte que l'une sans l'autre cloche, et
vacile, Toutesfois aujourdhuy (je ne scay si c'est par negligence, ou à

37. Canappe « Maistre Jean Canappe Docteur en Medecine au Lecteur Chirurgien Salut ", in Tables anatomiques du corps humain universel, 7.

38. Canappe, «Philiatros au Lecteur Salut», in Le quatriesme livre de la Therapeutique (1537), f. 2 ro. 
cause de la practique lucrative, à laquelle la plus grand part des medecins estudient plus que à la theorique, qui n'est autre chose sinon la parfaicte et entiere congnoissance des maladies, et temperatures des corps humains, avec les facultez et vertus des medicamens, dont est prise l'indication curative) on separe lesdictes parties de medecine, desquelles la premiere est demeurée à ceulx que le vulgaire appelle medecins. La seconde aux apoticaires, dont ilz retiennent le nom des pharmacopoles. Et la tierce est demeurée aux chirurgiens. ${ }^{39}$

Canappe propose ainsi de rétablir une perméabilité des savoirs et des pratiques, dans une perspective pleinement humaniste. Surtout, Canappe inscrit sa démonstration dans le cadre d'une réflexion sur le langage et ses fonctions. Certes, la médecine, en tant que théorie, équivaudrait à la philosophie, alors que la chirurgie et la science apothicaire, pratiques, correspondraient à l'éloquence. Mais par cette assimilation, Canappe ennoblit précisément ces parties dévalorisées. Il les associe surtout à une pratique du langage comme outil de transmission et comme vecteur d'un savoir (et non comme une fin en soi), légitimant ainsi une autre pratique du langage, celle de la traduction qu'il propose.

La traduction apparaît dès lors comme un enrichissement, puisqu'elle permet d'offrir ce qui n'existe pas encore en français en ouvrant le champ des savoirs vernaculaires. C'est ainsi que Canappe justifie son choix de traduire l'ouvrage d'anatomie de Louis Vassé : "Pource que nous n'avons point d'anatomie en nostre langue assez ample, et suffisante : ains mutilée, imparfaite, et souventesfois mal consonante à vérité ${ }^{40}$. Surtout, elle est le lieu d'une revendication idéologique des capacités de la langue française à véhiculer toute science, contre une supériorité supposée des langues anciennes. La démarche de Canappe et de Tolet s'inscrit dans le travail de défense et d'affinement de la langue française qui se développe à Lyon à partir de la fin des années 1530, initialement dans l'officine de François Juste, puis chez Dolet et enfin Jean de Tournes. Pierre Tolet est particulièrement clair à ce sujet et s'engage pour la promotion des langues vulgaires au premier rang desquelles devrait figurer

39. Canappe, «Philiatros au Lecteur Salut», in Le quatriesme livre de la Therapeutique (1537), f. $2 \mathrm{r}^{\circ}-\mathrm{v}^{\circ}$. 40. Canappe, "Maistre Jean Canappe Docteur en Medecine au Lecteur Chirurgien Salut », in Tables anatomiques du corps humain universel, 6. 
le français. Après avoir justifié sa traduction des traités de Paul d'Égine et de Galien par la réponse apportée à la demande des chirurgiens lyonnais, il ajoute :

L'autre (et la principale) a esté, pour ce que maintenant plusieurs Autheurs antiques, et modernes, sont illustrés, et publiés par nostre langue vulgaire. Et non seulement cecy se faict en France, mais en tous aultres Royaulmes, et contrées. Pour exemple, nous voyons les Italiens reduire en langue Italienne presque tous les Autheurs Latins, et Grecz : et se delecter plus de leur langue naturelle, que de toute aultre estrangiere, et peregrine. Semblable chose faict l'Espaignol : lequel non content de ses histoires vulgaires, et autheurs composez en sa langue, a traduict à la fin Avicenne de la langue arabicque en Espaignole. Je laisse les Allemans, Angloys, Escossoys, et les Grecz vulgaires : lesquelz a present sont contentz de la lecture de leur seulle langue maternelle. Et non pour ce me sera objectée l'abolition de la langue Latine, ou Grecque artificielle : lesquelles ne doibvent estre familieres, et communes à ung chascun. ${ }^{41}$

Pour Tolet, plus explicitement que pour Canappe à notre sens, l'utilité de la traduction pour les chirurgiens n'est pas la principale motivation du translateur. Le travail de Tolet sur Paul d'Égine et Galien s'inscrit dans une entreprise plus vaste menée en particulier par Dolet, que Valerie Worth-Stylianou a contribué à mettre en lumière ${ }^{42}$. À cet égard, d'après une épigramme de Charles de Sainte-Marthe jouant sur la quasi-homophonie des patronymes, Tolet et Dolet sont particulièrement intimes et partagent les mêmes aspirations ${ }^{43}$. C'est particulièrement visible dans la dédicace de sa traduction de Villegagnon, que Tolet offre à Jean du Peyrat :

[...] si les langues doctes ausquelles il y a grand artifice et par lesquelles les haultz dictz sont gardez et cachez aux vulgaires par bonne diligence se viennent à apprendre comme feist un jadis nommé Budée lequel se rendit la langue grecque si familière que Gaza et Lascaris Grecqz natifz n'eussent

41. Tolet, " Proesme au Chyrurgien Francoys », 3-4.

42. Worth-Stylianou, «Étienne Dolet et l'édition médicale », 361-379.

43. Charles de Sainte-Marthe, La Poësie Françoise de Charles de Sainte Marthe natif de Fontevrault en Poictou. Divisée en trois Livres. Le tout addressé à tresnoble et tresillustre, Princesse Madame la Duchesse d'Estampes et Comtesse de Poinctievre. Plus, Un Livre de ses Amys (Lyon : Le Prince, 1540), fol. A 6 rº 11. 
sceu mieulx escripre quelque inclination naturelle qui y fust, par plus grande raison doncques, singulier Seigneur, pensera le detracteur que l'on pourra faire aux langues vulgaires faisant fin. ${ }^{44}$

Très clairement, l'entreprise de Tolet n'est pas strictement médicale. Elle s'adresse également aux amis traducteurs tels qu'Étienne Dolet, Jean Des Gouttes, Charles de Sainte-Marthe, Charles Fontaine ainsi que d'autres traducteurs à venir, encore anonymes. Elle vaut surtout comme prélude à des publications médicales directement rédigées en français, comme il l'accomplit, effectivement, en 1549, avec le Paradoxe de la faculté du vinaigre :

[...] joint que aussi bien l'on peult ratiociner en François, ou autre langue vulgaire, que en Latin. Marsilius Ficinus Florentin, homme en Grec et Latin admirable, ha bien escrit en langage Italien les preceptes et remedes contre la Pestilence, et depuis ha esté mis en Latin par un docte Allemant nommé Hieronymus Ricius. ${ }^{45}$

On sait que les théories de la traduction développées par Tolet rejoignaient celles de Dolet dans La Manière de bien traduire ${ }^{46}$. Tolet conjugue un respect du texte original, dans une perspective philologique, et la conscience d'une nécessaire intervention ponctuelle du traducteur afin de favoriser l'intelligibilité du texte :

Saiches amy, qu'en traduisant le sixiesme Livre de Chyrurgie de Paulus Ægineta docteur exellent, et approuvé par les Medecins modernes (lequel toutesfois je ne tiens au nombre des anciens medecins) j'ay trouvé grande perplexité, et certain langaige amphybologicque. Ce que ne pense proceder

44. Pierre Tolet, "A messire Jehan du Peirat, conseiller du Roy, nostre Sire, Lieutenant-General de la Seneschaulcée de Lyon, Pierre Tolet ", in L'expedition et voyage de Lempereur Charles le quint en Affricque : Contre la Cité de Arges. Traduyte de latin en francoys par M. Pierre Tolet Medecin Lyonnoys (Lyon : Pierre de Sainte-Lucie, 1542). Traduction de : Caroli V Imperatoris expeditio in Africam ad Argieram per Nicolaum Villagagnonem equitem Rhodium Gallum. Ad D. G. Bellaium, Langæum, Subalpinarum Gentium proregem et primi ordinis Equitem apud christianissimum Francorum regem (Paris : Jean-Louis Tiletan, 1542).

45. Pierre Tolet, Paradoxe de la faculté du vinaigre (Lyon : Jean de Tournes, 1549), « Au lecteur chirurgien salut », 10 .

46. Élise Rajchenbach, Mais devant tous est le Lyon marchant. Construction littéraire d'un milieu éditorial et livres de poésie française à Lyon (1536-1551) (Genève : Droz, 2016), 66-68. 
de l'autheur, mais de l'interpreteur Latin : combien que l'autheur ayt voulu estre brief, et eviter toute prolixité de langaige. Le malheur doncq est tel, que les interpreteurs du temps present sont tant astrainctz aux parolles, que plustost ilz accommodent les choses au parler, que les parolles aux choses. Parquoy bien souvent confundent, et depravent le sens de l'autheur, tellement que ny l'autheur, ny l'interpreteur sont entendus. Voulant doncq mectre les choses en lumiere, et eviter toute obscurité, aux lieux plus difficiles, tant pour les noms des maladies, que les noms des instrumentz Chyrurgicaux, que l'appellation de la partie malade, pour plus facile intelligence ay entrelassé quelque declaration mienne, oultre la letre de l'autheur. Et trouveras fermé, ce qui est nostre, dedans deux demis quadratz de ceste figure [ ]. ${ }^{47}$

Suivant les préceptes développés par Dolet dans La Manière de bien traduire, Tolet favorise l'idée sur la lettre et, dans un souci philologique d'une grande rigueur, il signale entre crochets ses interventions sur le texte. En ce sens, il participe, par l'exemple, au travail de régulation des principes de la traduction fidèle et rigoureuse. La rigueur traductrice, indispensable, pour les textes médicaux, à la survie du patient, est étroitement liée à la rigueur philologique ainsi qu'au souci de transmettre un texte qui soit le plus complet possible, comme en témoigne l'adjonction, in extremis, d'un livre retrouvé. Rabelais le rappelait avec fermeté dans la préface de son édition d'Hippocrate et de Galien :

Id quod si usquàm alibi vitio verti solet, est etiam in medicorum libris piaculare. In quibus vocula unica, vel addita, vel expuncta, quin et apiculus inversus, aut præpostere adscriptus, multa hominum millia haud raro neci dedit. ${ }^{48}$

47. Tolet, « Proesme au Chyrurgien Francoys », 12.

48. Cité et traduit par Antonioli, Rabelais et la médecine, 81 : «Si partout ailleurs, on voit là des fautes, ce sont bien, dans un livre de médecine, des crimes car, dans ce cas, l'adjonction ou la suppression d'un simple mot, le changement d'un simple signe ou son déplacement ont plus d'une fois voué des milliers d'hommes à la mort ». Cité d'après l'éd. Gryphe de 1542, Aphorismorum Hippocratis sectiones septem, Ex Franc. Rabelosi recognitione. Quibus ex Ant. Musa Commentariis adjecimus et Octavam : et quadam alia, quoe sequens indicabit pagella, 4. 
C'est en ce sens que l'entreprise de traduction des textes galéniques s'inscrit surtout dans l'entreprise de conquête linguistique et politique du français. À cet égard, le choix même, par Tolet, de faire figurer sa défense et illustration du français en tête du texte de Villegagnon, c'est-à-dire d'une traduction retraçant la défaite terrible devant Alger de Charles Quint, l'éternel rival de François Ier, souligne bien la convergence des enjeux politiques et linguistiques. On comprend ainsi que ces traductions de textes médicaux rayonnent bien au-delà d'un simple public médical de barbiers et de médecins. Elles sont à réinscrire dans un mouvement plus vaste, commencé à la fin des années 1530, qui vise à ancrer le Royaume de France comme puissance linguistique, culturelle, scientifique et politique, bref, à en légitimer les aspirations européennes.

\section{Conclusion}

Les traductions médicales qui se développent à la fin des années 1530 et au début des années 1540 à Lyon s'adressent à un public parcouru de tensions, plus divers que le premier lectorat affiché. Si elles visent dans un premier temps à pourvoir les chirurgiens d'un savoir nécessaire au bon exercice de leur art, elles s'inscrivent surtout dans les débats qui agitent le milieu médical dans ces années. Elles rendent compte de l'écart qui sépare médecins et chirurgiens, et surtout des querelles qui agitent le groupe peu uniforme des médecins. De manière symptomatique, les traductions sont effectuées par deux médecins formés à Montpellier, c'est-à-dire dans une université progressiste, et qui fréquentent par ailleurs les cercles qui promeuvent la traduction à Lyon. En ce sens, elles s'inscrivent in fine au cœur d'une conquête proprement humaniste des savoirs et de leur diffusion, fondée sur la rigueur philologique, les réflexions sur la manière de bien traduire et les débats sur la place des langues vernaculaires. C'est en ce sens que Tolet présente son choix de traduire et d'écrire en français comme un choix guidé par une volonté de progrès qui touche les corps les plus divers : "Unde indoctis prodesse, et doctos delectare summopere puto, manumque hærentibus porrigere volo $»^{49}$. 


\section{Annexe Corpus de l'étude}

Ce corpus n'est pas exhaustif. Toutes les références de ces ouvrages sont faites dans les mêmes éditions.

Le quatriesme livre de la Therapeutique ou methode curative de Claude Galien, prince des Medecins, auquel est singulierement traictée la cure des ulceres, translaté par Philiatros (Lyon : François Juste, 1537) : dédicace du traducteur au lecteur.

Le Cinquiesme livre de la methode therapeutique de Claude Galien (Lyon : François Juste, 1537).

Quatorzième livre de la méthode thérapeutique (Lyon : Guillaume de Guelques, 1538).

Le Guidon en Françoys nouvellement reveu, et au vray corrigé, par maistre Jehan Canappe Docteur en Medecine, selon le jugement de plusieurs aucteurs anciens, comme Hippocrates, Galien, Avicenne, et autres: Avec la traduction du latin delaissé en toutes les autres Impressions : et Additions de plusieurs passaiges omis par le premier translateur notées a tel signe ${ }^{\star}$ Et aussi une Table faisant mention des matieres principales dudict livre (Lyon : Guillaume de Guelques, 1538) : dédicace au lecteur.

Le Troisiesme Livre de la Therapeutique ou Methode curatoire de Claude Galien prince des Medecins, auquel est singulierement traictée la cure des Ulceres [trad. Tolet] (Lyon : Guillaume de Guelques, 1539) : dédicace de Dolet à Canappe.

Le quatriesme livre de la Therapeutique, ou Methode curative de Claude Galien, prince des Medecins, auquel est singulierement traictée la cure des ulceres malingtz, translaté au vray par Philiatros (Lyon: Guillaume de Guelques, 1539) : dédicace au lecteur.

Le tresiesme Livre de la Methode Therapeutique de Claude Galien (Lyon : Guillaume de Guelques, 1539) : dédicace au lecteur.

La chirurgie de Paulus Agineta. Qui est le sixiesme livre de ses Euvres. Item ung Opuscule de Galien, des tumeurs contre nature. Plus ung Opuscule dudict Galien, de la maniere de curer par abstraction de sang. Le tout traduict en Françoys par Maistre Pierre Tolet Medecin de l'Hospital de Lyon (Lyon : 
Étienne Dolet, 1540) : dédicace «A Monsieur Squironis » et « Proesme au Chyrurgien Françoys".

Du Mouvement des muscles, Livre deux. Autheur Galien. Nouvellement traduict de Latin en Françoys, par Monsieur Jehan Canappe Docteur en Médecine (Lyon : Étiennne Dolet, 1541) : dédicace en latin à G. Rondelet et en français au lecteur (rééd. Sabon, 1556).

L'anatomie des os du corps humain, autheur Galien; nouvellement traduicte de latin en francoys, par monsieur Jehan Canappe (Lyon : Étienne Dolet, 1541) : dédicace aux chirurgiens.

Deux livres des simples de Galien. Le V. et le IX. Traduictz par Monsieur maistre Jehan Canappe, Docteur en Medecine (Lyon : Étienne Dolet, 1542) : dédicace au lecteur.

Des tumeurs oultre le coustumier de Nature. Opuscule nouvellement traduict de Grec en Latin : et de Latin en Francoys (Lyon : Étienne Dolet, 1542).

De la raison de curer par evacuation de sang. Autheur Galien (Lyon : Sulpice Sabon pour Antoine Constantin, s.d.) : dédicace au lecteur.

Tables anatomiques du corps humain universel : soit de l'Homme, ou de la Femme. Premierement composees en Latin par maistre Loys Vassee : Et depuis traduites par Jean Canappe (Lyon : Jean de Tournes, 1547) : dédicace au lecteur chirurgien. 